\title{
AC 2008-680: TALES FROM THE WAVE FRONT: TEACHING THE PHYSICS OF CELL PHONES AND WIRELESS COMMUNICATIONS
}

Robert Ross, University of Detroit Mercy

Jenna Ross, Utica Center for Mathematics Science and Technology 


\section{Tales from the Wave Front: Teaching the Physics of Cell Phones and Wireless Communications}

\section{Introduction}

For over twenty years our college has offered a summer enrichment program for high school students. The UNinitiates Introduction to Engineering (UNITE) program affords high school students the opportunity to take courses in writing, mathematics, computer science, persuasive speaking, and physics. Our unique program targets incoming high school freshmen from a diverse urban population. For several years the physics course was based on a traditional introductory college mechanics laboratory curriculum. This curriculum was not inquiry-based and provided only limited opportunities for students to construct their own knowledge by performing open-ended activities.

Scholarly research into the teaching and, more importantly, the learning of physics has provided valuable guidance for the design of innovative curricula and pedagogy ${ }^{1}$. The pedagogical strategies that are able to demonstrate high rates of student achievement, as measured by standardized examinations, involve some form of what is commonly called interactive engagement. Hake defines, "Interactive Engagement', (IE) methods as those designed at least in part to promote conceptual understanding through interactive engagement of students in heads-on (always) and hands-on (usually) activities which yield immediate feedback through discussion with peers and/or instructors,..." A variety of instructional methods such as collaborative learning, peer instruction, tutorials, and computer based instruction have been developed to promote conceptual understanding (a comprehensive review of the literature can be found in reference 1). In addition to the method of instruction, students bring their personal histories with them to class, and it has been shown that their expectations play a critical role in the outcome of a physics course ${ }^{3}$. The interactive engagement methods, which have demonstrated success, rely on a constructivist approach to learning. Learners "construct" their own knowledge as a result of experiences provided by the instructor and the curriculum.

For the past two years we have used the Physics of Cell Phones and Wireless Communications, an innovative, hands-on, inquiry-based curriculum ${ }^{4}$. Other innovative science and engineering programs have been developed using the constructivist approach to enrich the learning experiences of high school students ${ }^{5}$. The Physics of Cell Phones and Wireless Communications curriculum was developed by high school physics teachers to comply with state curriculum standards and guidelines in the natural sciences. The content was designed to be covered in a high school physics classroom over a four week period. We find that in addition to being well suited to our UNITE high school enrichment program, the curriculum offers an additional benefit; many of the activities are appropriate for demonstrations and laboratory exercises in an introductory university physics course. University students respond favorably to the introduction of state-of-the-art technology ${ }^{6}$. The curriculum is relatively inexpensive and traditional laboratories have much of the necessary additional support equipment. We will describe this curriculum from both the faculty and student points of view and discuss how we adapted this curriculum to fit the needs of our individual program. We will highlight some of the activities that are suitable for university level work. 


\section{Curriculum}

The Physics of Cell Phones and Wireless Communications curriculum consists of 24 studentcentered activities in support of 30 well-articulated learning outcomes. We note that the activities are broadly grouped in 3 categories: the properties of sound and light waves, analog and digital signals and their transmission, and cellular telephone network operations. We focused on the first two categories and omitted some of the material regarding network operations. It should be noted that the network operations portion of the curriculum is interesting as it provides hands-on, interactive activities to simulate Time Division Multiple Access (TDMA), Code Division Multiple Access (CDMA), and Global Systems Mobile (GSM) methods. The activities are in the form of a role-playing game where students model cell connections and handoffs. It is quite possible that these activities are appropriate for upper-level electrical engineering students in a communications course.

The Physics of Cell Phones and Wireless Communications package contains equipment for 24 students with the expectation that the activities and experiments will be performed in groups. The students can do much of the work at their own pace while some sets of activities need to be coordinated by splitting the class into two groups. Each student receives a reusable instruction manual and a set of laboratory notes to be completed during class. The bundle includes eight kits; each with an audio amplifier, AM-FM radio, encapsulated silicon solar cell, audio speaker, and appropriate cables. In addition to the eight kits, there are four sets of walkie-talkies, light emitting diode (LED) bias circuits, fiber optic cables, laser pointers, springs and weights, and manipulatives for modeling a cellular network. The authors of the curriculum assume that the laboratory is equipped with induction coils and a simple computer based data acquisition system for graphing current or voltage as a function of time. A photograph of some of the equipment is shown below in Figure 1.

We describe some of the activities and experiments completed by students. We are providing only a general description, not necessarily in the order in which they were performed.

\section{Wireless Telecommunication}

One of the first activities involves a wireless form of communication. Students connect the output of the radio to an induction coil. A second induction coil is then connected to the audio amplifier. When the two coils are in close proximity, the audio output of the radio can be heard coming from the amplifier. Students have an opportunity to vary the position and relative orientation of the two coils to determine what affects the sound. Of course the coupling between the coils is inductive and the signal is not propagated via an electromagnetic wave. Subsequently, students construct a primitive wireless telephone system with the audio speaker used as a microphone. The output of the speaker is fed into an amplifier; the output of the amplifier is directed, once again, into an induction coil. The receiver coil is connected to another audio amplifier. By talking into the speaker, students can hear their voice from the audio amplifier. They are asked to complete worksheet where, amongst other things, they describe the energy transformations involved in the communication process. 


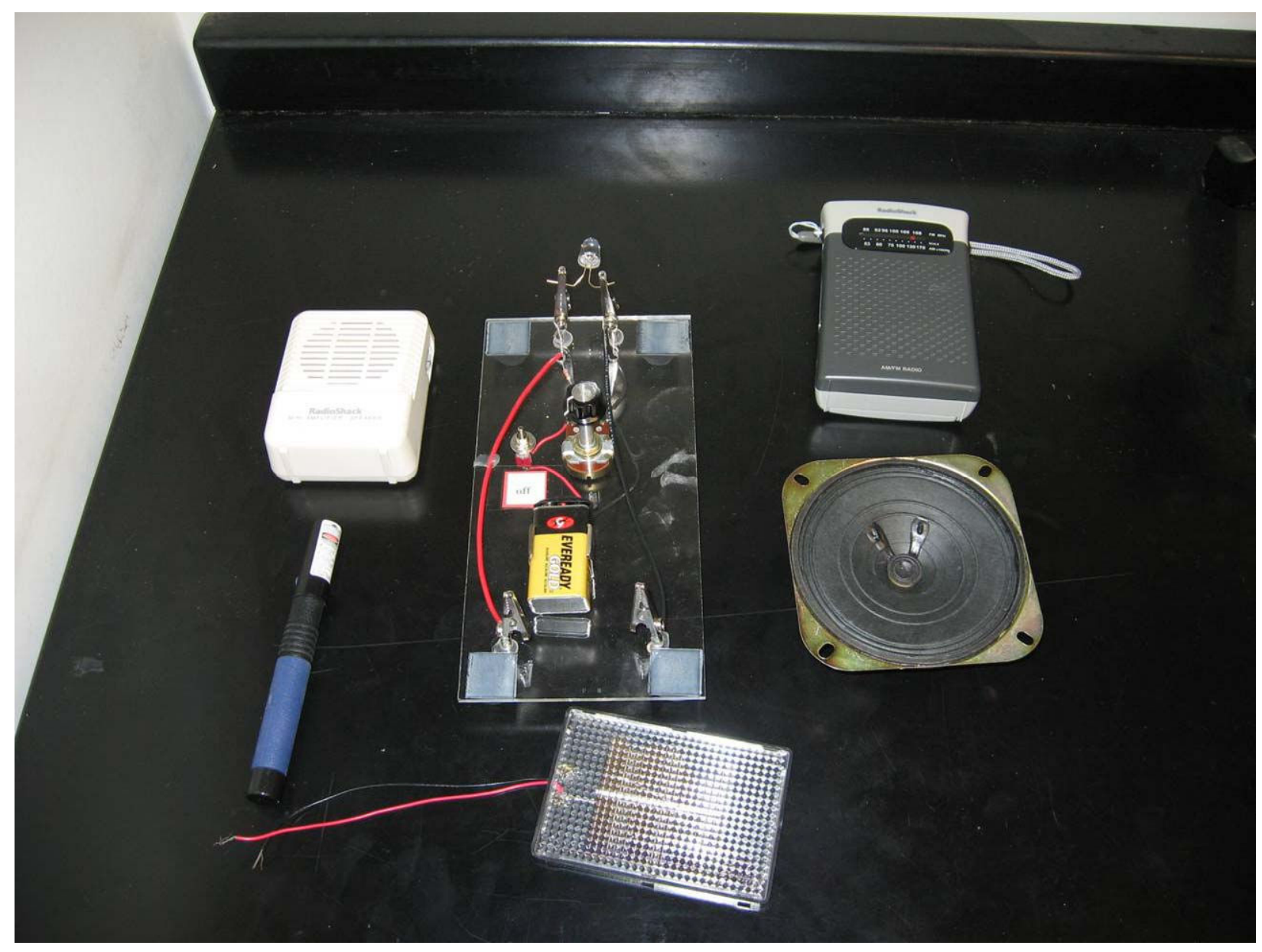

Figure 1. The photograph shows the audio amplifier, LED bias circuit, AM-FM radio, laser pointer, solar cell and audio speaker included in the kits.

The kit includes a light-emitting diode (LED) bias circuit. The output of the AM-FM radio is connected to a biased LED. The LED subsequently flashes with the same frequency as the audio portion of the radio signal. The silicon solar cell is connected to the audio amplifier. When the LED illuminates the solar cell, the audio output of the radio can be heard coming from the amplifier. The setup is shown below in Figure 2. It should be noted that this works over distances of several meters. 


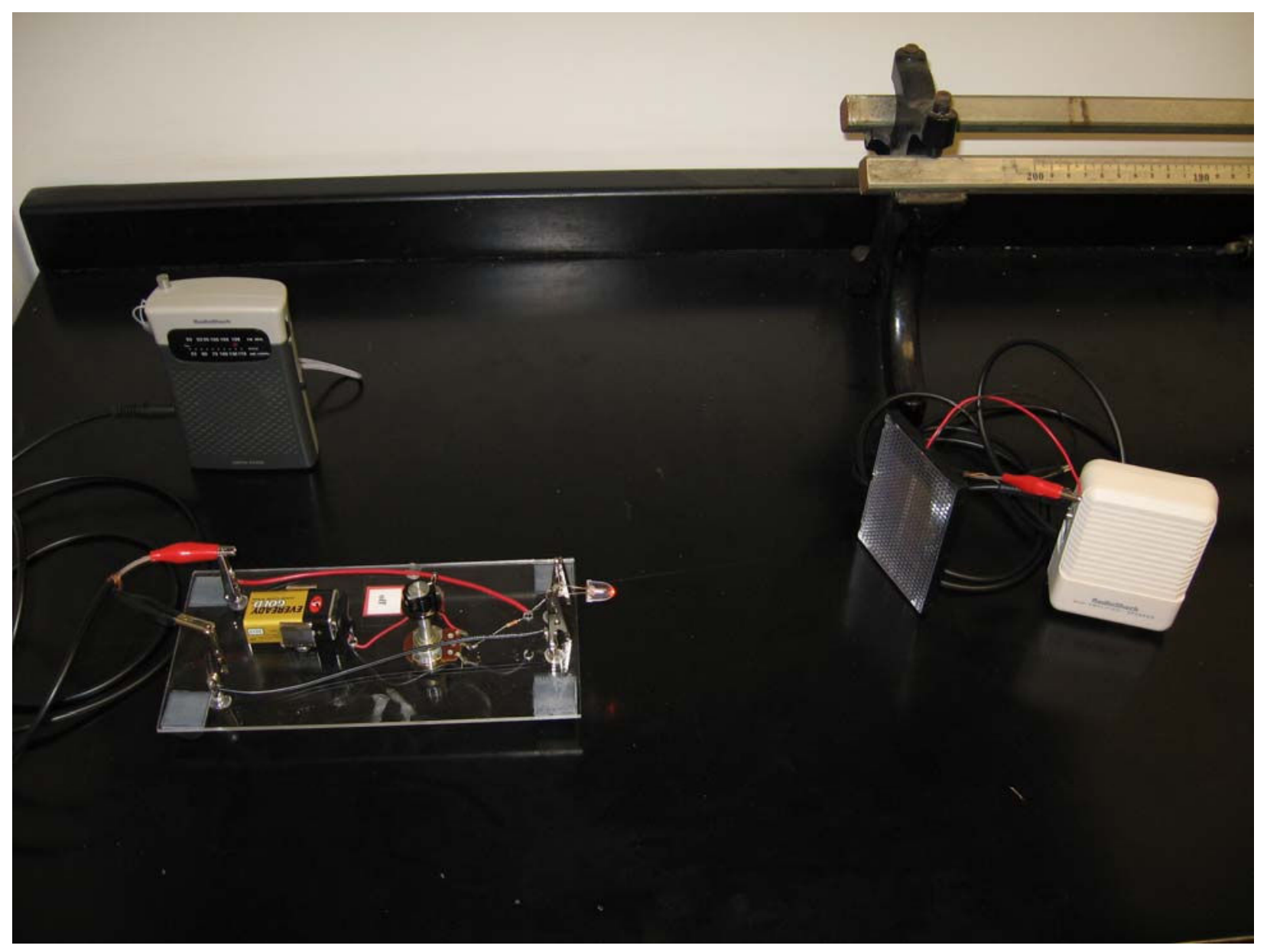

Figure 2. An LED bias circuit used to wirelessly transmit audio information.

Students then use an optical fiber to connect the light source to the detector. In this configuration the LED source and silicon detector do not need to be in alignment. This situation is shown below in Figure 3. Students of all levels are quite interested in these activities and we have been using them in our introductory courses. 


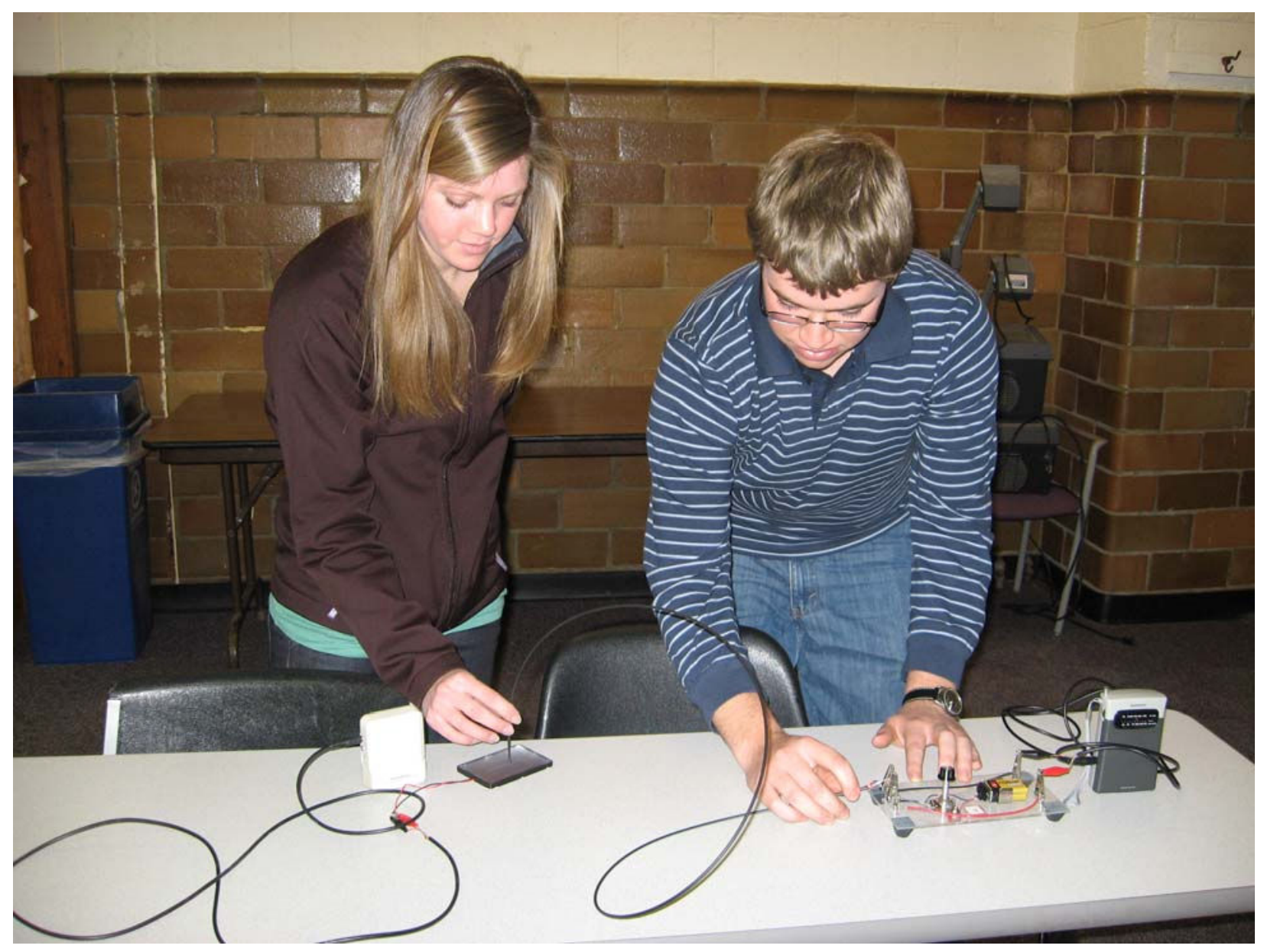

Figure 3. An optical fiber is used to transmit information from the LED bias circuit.

\section{Cells}

The activity to investigate the shape of a "cell" in a cellular telephone network is useful for a wide range of students. From the beginning of the program, students are encouraged to observe any cell phone towers they see around them and to take pictures if they have a camera. Of course they always seem to have a telephone handy with a digital camera. When the students compare the pictures of the towers they recognize that each tower has a triangular shape on the top. The towers radiate in three directions, $120^{\circ}$ apart. Students use manipulatives to investigate the shape of the cells in the network. They find that only a few regular geometric shapes can tile a surface without any gaps in coverage. Using this information, along with the triangular antennae, leads students to conclude that the cells in a cellular network are hexagonal and the antennae are located at the intersections of three adjacent hexagonal cells. Figure 4. shows the manipulatives used in the exercise. We use this simple activity in our introductory laboratory and find that university students are generally surprised to discover that the cell towers are not in the center of a cell. 


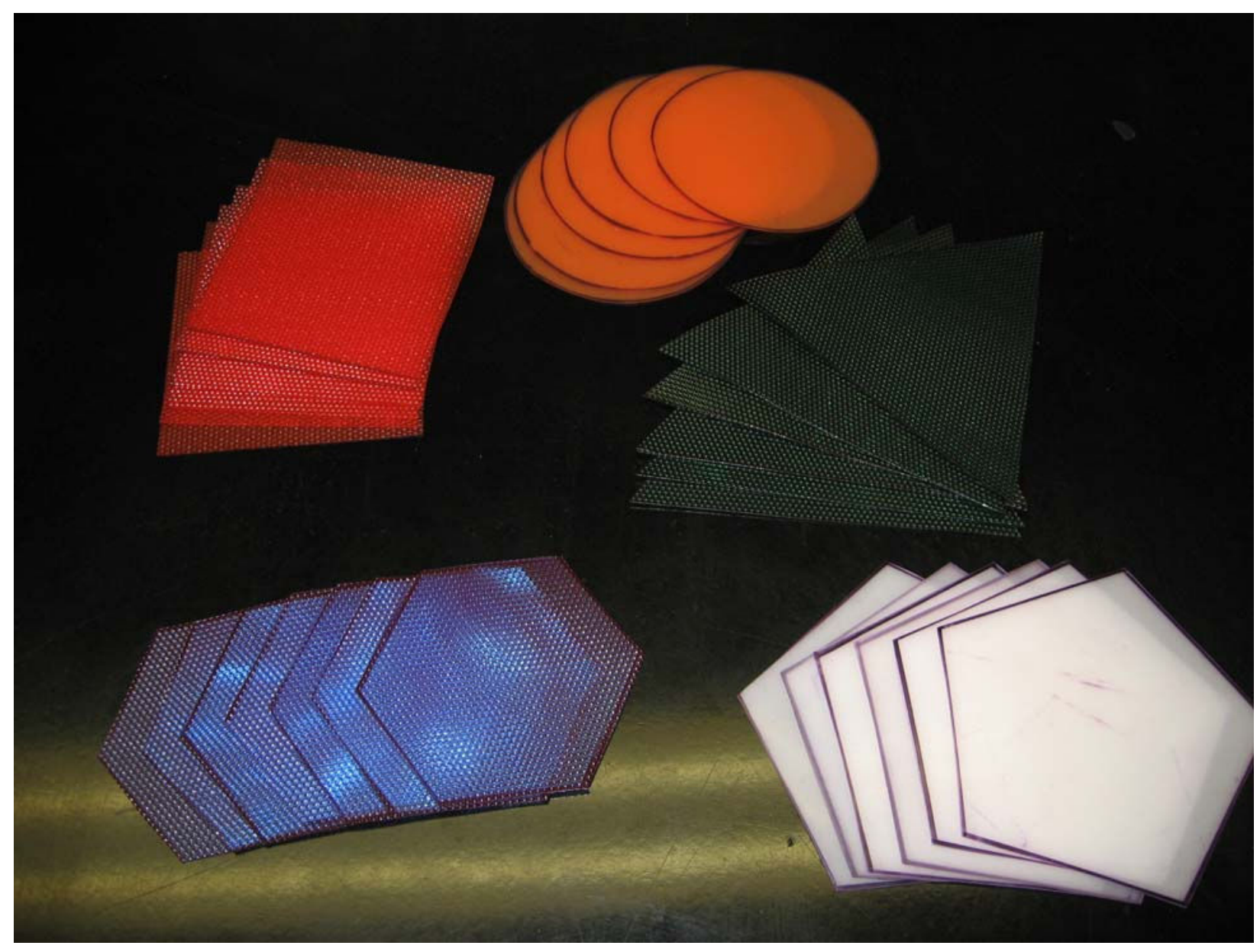

Figure 4. One set of manipulatives used to investigate the shape of cells in a cellular network.

\section{Sound}

The audio speaker is used as a microphone and the amplifier boosts the signal enough to be read by an inexpensive computer data acquisition system. We use the Pasco Xplorer system with the current-voltage probes and the associated Pasport software. The voltage waveform can be displayed on the monitor and students can distinguish between pitch and amplitude for the sounds they generate. The software has a fast Fourier transform function that displays the frequency components. We modified this activity by using tuning forks in addition to the student's voices. The amplitude can be reproducibly adjusted by varying the distance of the tuning fork to the speaker, this worked better than having the students try to increase the loudness without changing the pitch.

\section{Analog and Digital Signals}

Students perform a guided activity to digitize an analog waveform. They draw an arbitrary graph of voltage as a function of time. They digitize the function using a binary number table and record the information. They subsequently transfer the binary code to a partner who tries to reproduce the original waveform. The students recognize that more frequent sampling with 
greater voltage resolution leads to improved accuracy in reproduction. We felt it was valuable to add more content to the activity by introducing some elementary binary logic. Students were introduced to the AND, OR, NOT and XOR (exclusive-OR) logic functions. They used a self generated key, along with an ASCII (American Standard Code for Information Interchange) conversion table to encode and transmit an encrypted binary message to a partner. The partner used the encryption key to decode the message.

The curriculum affords students the opportunity to experience digital signal transmission in both audio and visual formats. When the solar cell is connected to the input of the audio amplifier, students can hear the temporal pattern of light, either visible or infrared, that is producing current in the cell. Room light causes audible noise to be generated by the amplifier. Students hear the oscillation due to the alternating current in the light. They hear a different pitch when they hold the cell up to the front surface of a computer monitor, which refreshes at $72 \mathrm{~Hz}$, and they hear deafening silence when the cell is placed in direct sunlight. Television remote controls use infrared LEDs to produce a digital signal. When one is directed at the solar cell and amplifier combination, sounds are heard as buttons are depressed. We use this activity to show that infrared light reflects from a mirror in the same manner as visible light. A photograph of two students experimenting with the remote control is shown in Figure 5.

In a subsequent activity, a laser pointer is directed at the solar cell and an ordinary comb is used to interrupt the light beam. When the comb is moved back and forth a distinct sawing sound is generated by the amplifier. The pitch varies as the speed of the "sawing" is changed.

If the output of the amplifier is connected to the data acquisition system the variations in current can be observed. Students use this to create a bar code scanner. A section of an overhead transparency is covered with opaque masking tape in a pattern representing a binary code. When the transparency is swept though the beam of the laser pointer, a pattern of current is displayed on the screen. The pattern of current as a function of time contains the binary information encoded on the transparency.

\section{Student Comments and Observations}

A sample of some student comments and observations are reported below.

"To high school students, the cell phone has become a necessity. Our dependency to have constant contact with people we know, has led to almost every one of us having our own phones. For this reason, when we went into a classroom and learned all about the inner workings of cell phones, we were much more eager to learn as much as we could."

"We have been curious as to how signals travel without wires, why those huge towers are everywhere in the city, and what causes the dreaded "signal faded" message on our cell phone screens. This program answered our questions and put an end to our curiosities."

"The course was designed to be very hands-on, which was a good way to keep our interest." 
"Hearing the sounds, seeing the lights, feeling the vibrations, and reading the data on the graphs was a much better way to learn, and a much more interesting way as well. We enjoyed every minute of the experience."

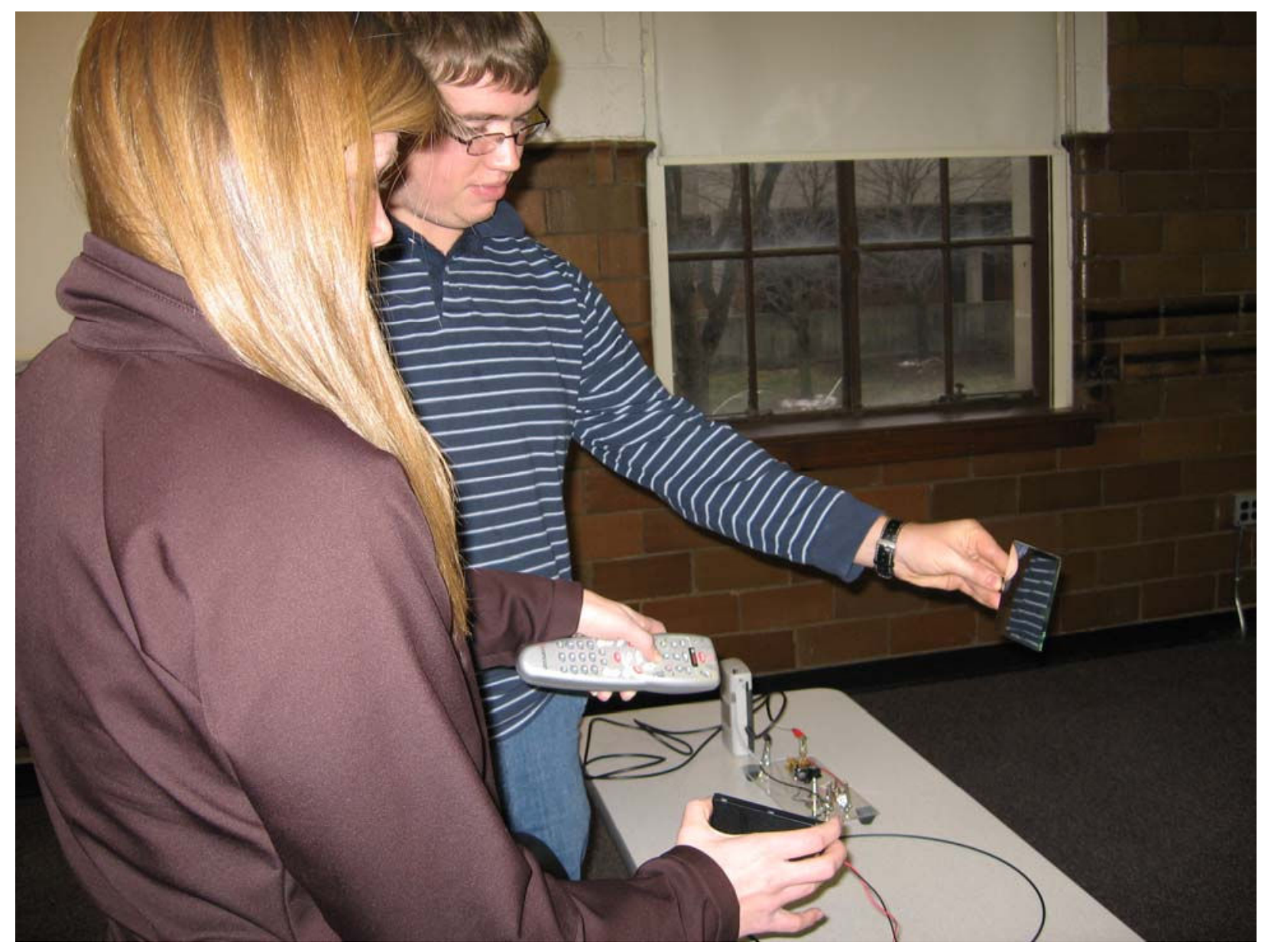

Figure 5. Using a remote control to demonstrate the reflection properties of infrared light.

\section{Conclusions}

Cellular telephones and other wireless communication devices are ubiquitous. We believe that important concepts in physics and electrical engineering can be taught in the context of common wireless communication devices and we find the Physics of Cell Phones and Wireless Communications to be well suited for this. Our experience with the curriculum leads us to conclude that students are motivated and engaged in a process of constructing their own knowledge of common wireless technology. We have identified two broad areas where the Physics of Cell Phones and Wireless Communications is useful. It appears to motivate high school students to study physics and engineering, at least during the class. Second, many of the activities are suitable for an introductory physics course at the university level. 


\section{Bibliography}

1. For a comprehensive review see, L.C. McDermott, and E.F. Redish, "Resource Letter: PER-1: Physics Education Research", Am. J. Phys., 67 (9), September 1999, pp. 755-767, and references therein.

2. R.R. Hake, "Interactive-engagement versus traditional methods: A six-thousand-student survey of mechanics test data for introductory physics courses", Am. J. Phys., 66 (1), January 1998, pp. 64-74.

3. E.F. Redish, J.M. Saul, and R. N. Steinberg, "Student expectations in introductory physics", Am. J. Phys., 66 (3), March 1998, pp. 212-224.

4. M. Davids, R. Forrest, and D. Pata, "Physics of Cell Phones and Wireless Communications," (2005).

5. W. Hwang, A. Bissell, D. Kaplan, M. Mian, V. Agrawal, J. Manson, and G. Ybarra, "Design and Evaluation of InnoWorks: A Portable, Interdisciplinary Science and Engineering Program by Volunteer College Students for Middle School Youth from Underprivileged Backgrounds", Proceedings of the 2006 American Society of Engineering Education Annual Conference \& Exposition, Chicago, IL (2006).

6 D.R. Loker, R.P. Krahe, J. Kirsch, T.J. Yowonske, R.J. Cunningham, and J.R. Petrovich, "Remote Sensing with GPS Sensor and Cellular Modem", Proceedings of the 2004 American Society of Engineering Education Annual Conference \& Exposition, Salt Lake City, UT (2004). 